OPEN ACCESS

Edited by:

Yan Mark Yufik,

Virtual Structures Research, Inc.,

USA

Reviewed by: Alianna JeanAnn Maren Northwestern University, USA Paul John Werbos, Retired, USA

*Correspondence: Robert Kozma rkozma@memphis.edu

Received: 07 October 2016 Accepted: 16 February 2017 Published: 14 March 2017

Citation:

Kozma $R$ and Freeman WJ (2017) Cinematic Operation of the Cerebral Cortex Interpreted via Critical Transitions in Self-Organized Dynamic Systems.

Front. Syst. Neurosci. 11:10. doi: 10.3389/fnsys.2017.00010

\section{Cinematic Operation of the Cerebral Cortex Interpreted via Critical Transitions in Self-Organized Dynamic Systems}

\author{
Robert Kozma ${ }^{1,2 *}$ and Walter J. Freeman ${ }^{3}$ \\ ${ }^{1}$ College of Information and Computer Sciences, University of Massachusetts, Amherst, MA, USA, ${ }^{2}$ Department of \\ Mathematical Sciences, University of Memphis, Memphis, TN, USA, ${ }^{3}$ Department of Molecular and Cell Biology, University of \\ California at Berkeley, Berkeley, CA, USA
}

Measurements of local field potentials over the cortical surface and the scalp of animals and human subjects reveal intermittent bursts of beta and gamma oscillations. During the bursts, narrow-band metastable amplitude modulation (AM) patters emerge for a fraction of a second and ultimately dissolve to the broad-band random background activity. The burst process depends on previously learnt conditioned stimuli (CS), thus different AM patterns may emerge in response to different CS. This observation leads to our cinematic theory of cognition when perception happens in discrete steps manifested in the sequence of AM patterns. Our article summarizes findings in the past decades on experimental evidence of cinematic theory of cognition and relevant mathematical models. We treat cortices as dissipative systems that self-organize themselves near a critical level of activity that is a non-equilibrium metastable state. Criticality is arguably a key aspect of brains in their rapid adaptation, reconfiguration, high storage capacity, and sensitive response to external stimuli. Self-organized criticality (SOC) became an important concept to describe neural systems. We argue that transitions from one AM pattern to the other require the concept of phase transitions, extending beyond the dynamics described by SOC. We employ random graph theory (RGT) and percolation dynamics as fundamental mathematical approaches to model fluctuations in the cortical tissue. Our results indicate that perceptions are formed through a phase transition from a disorganized (high entropy) to a well-organized (low entropy) state, which explains the swiftness of the emergence of the perceptual experience in response to learned stimuli.

\footnotetext{
Keywords: cinematic theory of cognition, AM pattern, criticality, phase transition, Freeman $\mathrm{K}$ set, Hebbian assembly, graph theory, neuropercolation
}

\section{INTRODUCTION}

It is now commonplace to regard cerebral cortex as an organ maintaining itself in a dynamic state at the edge of criticality (de Arcangelis et al., 2014; Plenz and Niebur, 2014). Criticality in mathematics and physics relates to a point of sudden transition from one state to another. In thermodynamics, the term denotes a point on the phase boundary between solid, liquid and gas phases. Near the critical point, the state of the system changes drastically with the variation of some control parameter, which behavior has been observed in the operation of the cortex 
(Freeman, 2008; Fraiman and Chialvo, 2012; Freeman et al., 2012). Metastability is a related fundamental behavior employed in characterizing brain dynamics and cognition (Bressler and Kelso, 2001; Freeman and Holmes, 2005; Tognoli and Kelso, 2014). Metastability indicates a continuous interplay between phase synchrony and phase scattering in a system with many interacting components (van Straaten and Stam, 2013; Zalesky et al., 2014; Freeman, 2015).

How does the cortex maintain a critical state? Nuclear physicists use the concept of criticality to denote the threshold, at which nuclear fission reaction is maintained. The critical state of the fission chain reaction is achieved by a delicate balance between the material composition of the reactor and its geometrical properties. The criticality condition is expressed as the identity of geometrical curvature (buckling) and material curvature. Critical processes in nuclear reactors are designed in a way to satisfy strictly linear operational regimes, in order to guarantee stability of the underlying coupled reactor dynamic process (Upadhyaya et al., 1980; Kozma, 1985; March-Leuba and Rey, 1993). In brains, however, nonlinear feedback effects are of primary importance in sustaining complex cortical dynamics (Kozma and Freeman, 2001; Tagliazucchi and Chialvo, 2012). Our answer to the question on the origin of sustained critical state in brains is that mutual excitation between populations of cortical neurons maintains criticality, in combination with the refractory period that prevents exponential grow, thus stabilizes the dynamics (Freeman, 1975, 2004a).

In the past decade, neuroscientists successfully employed the concept of self-organized criticality (SOC) to neural processes (Beggs, 2008; Friston et al., 2012; Fingelkurts et al., 2013; Palva et al., 2013; Plenz and Niebur, 2014). These and many other studies point to scale-free dynamics in the cortex resembling cascades of sand piles during metastable states (Bak, 1996; Jensen, 1998; Petermann et al., 2009). SOC, however, cannot describe the existence of robust critical regions with sustained metastable dynamics, neither the rapid transitions from one metastable state to the other (Tognoli and Kelso, 2014). Bonachela et al. (2010) describe brains as "pseudo-critical" and suggest that we should "... look for more elaborate (adaptive/evolutionary) explanations, beyond simple self-organization.” Reinforcement learning (RL) is crucial in producing rapid transitions from one metastable state to the other (Freeman, 1979). RL sensitizes the cortex selectively and creates spatially extended Hebbian cell assemblies (HCAs). Once HCAs are formed, they respond collectively to conditional stimuli. Stimulating any part of the assembly triggers a rapid increase in synaptic gain, leading to the explosive increase in the activity, until the activation density reaches saturation (Freeman, 2015). HCAs manifest emergent neural packets facilitating the understanding of perceptual experiences (Yufik and Friston, 2016).

Synchronized bursts of neural activity have been observed and analyzed extensively in the literature. This includes the description of spike bursts in interacting excitatory-inhibitory neural populations (see, e.g., Hindmarsh and Rose, 1984; Izhikevich, 2000; Coombes and Bressloff, 2005; Srinivasan et al., 2013). Mathematical models based on chaos theory have been proved to be useful to describe these bursts patterns
(Hansel and Sompolinsky, 1992; Tsuda, 2001; Kozma, 2003). Recent breakthroughs include the comprehensive description of sharp wave ripples representing episodic memory effects (Buzsáki, 2015) and systematic analysis of spike bursts (Werbos and Davis, 2016). Our work addresses experimental and theoretical findings of transient synchronization in mesoscopic neural populations and their interpretation based on the concept of phase transitions in random graph theory (RGT) and statistical physics.

Since the early 2000s, phase transition in RGT has been employed as a useful mathematical concept to model the dynamics of the cortical tissue (Kozma et al., 2001). The random graph description of the cortex, called "neuropercolation," implements a hierarchy of cortical models (Kozma et al., 2005). Non-local interactions between neural populations via long axonal projections are crucial in describing cortical dynamics. There are extensive studies to model small-world effects (Watts and Strogatz, 1998) in structural and functional brain networks tuned to criticality (Bullmore and Sporns, 2009, 2012; Turova, 2012; Haimovici et al., 2013; Sporns, 2013; Alagapan et al., 2016). The level of system noise, the ratio of non-local connections corresponding to long axons, and the strength of inhibitory effects are key variables that allow controlling the transitions between opposite phases (Kozma and Puljic, 2015). In the absence of non-local connections, diffusion-like effects dominate the spatio-temporal dynamics, which fall short of producing the required rapid cortical transitions. With the help of non-local connections, we were able to generate and maintain phase transitions exhibiting rapid transitions between synchronized and desynchronized phases (Puljic and Kozma, 2008, 2010; Kozma and Puljic, 2015).

Phase transitions between disordered and ordered neural states provide key insights to understand and interpret the observed cortical space-time neurodynamics. Disordered states are characterized by random dispersion of active and inactive sites, while the emergence of metastable amplitude modulation (AM) patterns signify more ordered states. In the disorganized phase, the individual microscopic neurons are loosely coupled, which facilitates them processing sensory information individually. In the organized phase, the neurons are strongly coupled into populations producing metastable macroscopic AM patterns (Freeman, 2014). Transitions from one AM pattern to the other produce a sequence of metastable cortical states, which can be viewed as neural correlates of cognitive activity in the framework of the cinematic theory of cognition (Freeman, 2006, 2007; Kozma and Freeman, 2016). The cinematic theory of cognition is related to the concept of perception occurring in discrete epochs (Crick and Koch, 2003), and to the model of pulsating consciousness manifested via neuronal activity packages (Yufik, 2013).

This essay summarizes our decades-long experimental and theoretical studies supporting the concept of the cinematic theory of cognition. We review the theory of criticality in the cerebral cortex based on self-organized dynamics of neural populations, manifested in the form of sequential phase transitions between metastable AM patterns. In our interpretation, phase transitions are responsible for the rapid responses to sensory stimuli 
observed in cognitive processing and for the emergence of our perceptual experiences according to the cinematic theory of cognition.

\section{CONSTRUCTING THE SELF-ORGANIZED PERCEPTION CYCLE}

\section{Metastable AM Patterns Manifest the Organized Phase of Cortical Dynamics}

From the variety of the available brain monitoring techniques, here we focus on recordings EEG and ECoG potentials. Intracranial experiments with electrode arrays over the cortex have been conducted in various laboratories, providing a window on the electrophysiological processes underlying brain functions (Freeman, 1975; Skarda and Freeman, 1987; Canolty et al., 2010; Panagiotides et al., 2011; Buzsáki et al., 2012). A state-of-art overview of brain imaging using EEG and ECoG monitoring techniques is given by Freeman and Quian-Quiroga (2013), including single trial experiments, high-density arrays, and spatio-temporal spectral analysis. More traditional Fourier analysis is often supplemented by Hilbert transform, which is especially beneficial in the characterization of rapidly changing, metastable activity patterns.

We illustrate the experimental results concerning the presence of highly organized metastable AM patterns and their intermittent collapse to a disorganized state using the example of rabbits, conducted in the Freeman neurophysiology laboratory at UC Berkeley (Freeman and Barrie, 2000). Rabbits were implanted with intracranial electrode arrays over their sensory cortices and trained using the well established, RL paradigm. In the experiment displayed in Figure 1, an ECoG array of $8 \times 8$ electrodes is fixed over the visual cortex of the rabbit. The measurement is $6 \mathrm{~s}$ long with a visual stimulus presented to the animal at time instant $t=3 \mathrm{~s}$; thus there is a $3 \mathrm{~s}$ pre-stimulus and a $3 \mathrm{~s}$ post-stimulus period. Figure 1, upper plot, shows the 64 ECoG traces filtered in the gamma band 30-36 Hz (Davis et al., 2013). There is a base level of background activity during the $3 \mathrm{~s}$ expectancy state without stimulus. During the $\sim 1 \mathrm{~s}$ interval following the stimulus several gamma bursts appear. Finally, after about $1 \mathrm{~s}$ following the stimulus (at time instants $>4 \mathrm{~s}$ ), the activity returns to the background state. The novelty of the results lies in the development of quantitative measures to characterize the sequence of metastable states, using various pragmatic information indices (Freeman, 2004a; Davis et al., 2013).

Using Hilbert transform for each of the 64 ECoG signals, complex valued analytic signals are obtained with amplitude and phase components. The analytic amplitude represents the power of the ECoG signal, while the phase can be used to monitor synchronization effects. In Figure 1, lower plot, the amplitudes of the 64 analytic signals are shown. In the pre-stimulus period, the amplitudes fluctuate at a low level, indicating a sustained, disorganized background activity. There are several beats during the $\sim 1 \mathrm{~s}$ period following the stimulus, which demonstrate intermittent bursts of power in the gamma band. These bursts signify the emergence of metastable AM patterns (for details, see Freeman, 1975, 2004a, 2014).

The existence of an AM pattern indicates that the cortical dynamics is constrained to a narrow attractor basin in response to a given stimulus. This is a highly structured (organized) state with significant coordination between the 64 ECoG channels. In spite of the individual differences between the ECoG channels, they have significant commonality in their behaviors; namely, they rise, reach a maximum, and decrease in synchrony. This means that the AM pattern is largely time-invariant during the $100-200 \mathrm{~ms}$ of its existence, although its overall intensity varies in time. The relevance of AM patterns in defining the cognitive state of the animal has been demonstrated by using AM patterns as classification tools to discriminate between stimuli (Freeman, 1979; Kozma and Freeman, 2001). The AM patterns provide us with an observation window to monitor the cognitive process using ECoG/EEG techniques. When the input is removed, the cortical dynamics is released from its constrained state, the AM pattern disappears, and the cortex returns to the disorganized, background state.

The AM patterns do not represent the input stimuli in any practical sense; rather they correspond to the meaning of the input. They continuously change during the life of the animal through a learning process, as a result of past experiences, present state and future goals of the subject. If a new stimulus does not match a previously learnt experience, the response of the cortex

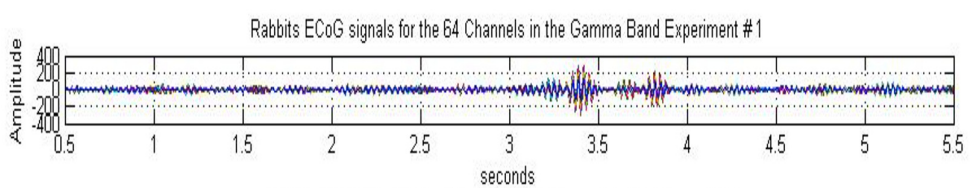

Rabbits ECoG Analitic Amplitud for the 64 Channels in the Gamma Band

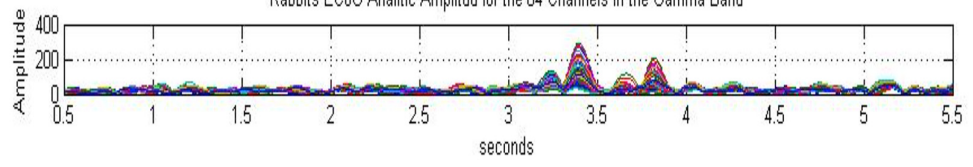

FIGURE 1 | Rabbit ECoG data measured over the visual cortex using an $\mathbf{8} \times \mathbf{8}$ array of electrodes. The duration of the experiment is 6 s, with a visual stimulus (light flash) presented to the animal at $t=3 \mathrm{~s}$; the signals were filtered over the gamma band (30-36 $\mathrm{Hz}$ ). The subplots show $64 \mathrm{curves}$ corresponding to the ECoG signals (top) and the analytic signals (bottom), respectively. The analytic signals have been calculated using Hilbert transform, from Davis et al. (2013). 
is a rapidly decaying oscillation. If the stimulus is presented again and again to the animal, the connections between excitatory neurons are strengthened in a process called Hebbian learning. As the result, the response decays less and less, which ultimately leads to sustained narrow-band oscillations due to the formation of a HCAs. The emergence of narrow-band oscillations is crucial for the efficient memory readout based on metastable AM patterns. The role of Hebbian reinforcement of connections between co-activated neurons has been demonstrated in large neuron populations, including the hippocampus, sensorimotor and speech areas (Buzsáki, 2005; Pulvermüller and Fadiga, 2010; Lopes-dos-Santos et al., 2013). In the computational domain, Hebbian RL has been implemented in various neural network models (see, e.g., Amit, 1995; Wennekers and Palm, 2009).

The example of the olfactory system with convergentdivergent connections is illustrated in Figure 2 (Freeman, 1979). Input is transmitted via the primary olfactory nerve (PON) to the olfactory bulb, where the HCA is shown by black dots. By stimulating any subset of the HCA, the whole HCA is activated and produces narrow-band oscillations, thus exhibits the key property of generalization over the category of the sensory stimulus. Activations from the bulb are projected to the olfactory cortex through the lateral olfactory tract (LOT). The increased strengths of mutual excitatory connections (Kee) in the Hebbian assembly strongly enhance gamma oscillations in response to learned stimuli (Baird et al., 1991; Kozma and Freeman, 2001). In the context of the present work it is to be emphasized that he formation of HCAs and their rapid activation in response to learned stimuli are important conditions of cortical phase transitions (Freeman, 2015).

\section{Background Activity and "Null Spikes"}

The low overall magnitudes of the ECoG and analytic signals in Figure 1 before the stimulus onset $(t<3 \mathrm{~s})$ indicate that the

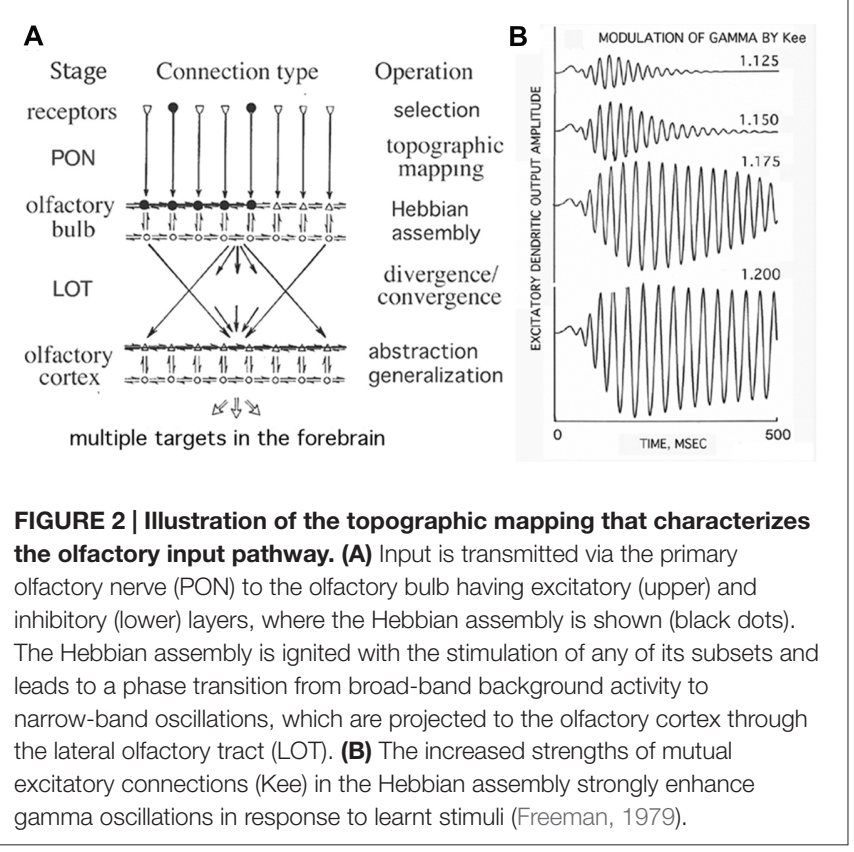

background activity is a state of relatively low energy as compared to the high-energy burst of the AM patterns. Moreover, the energy of the background oscillations is distributed over a wide range of frequencies as opposed to the narrow-band (gamma) oscillations contributing the formation of AM patterns. In fact, the background conforms to power-law dynamics with a power exponent ranging between -2 and -4 (Freeman and Zhai, 2009). It is generated by mutual excitation among populations of cortical excitatory neurons, which activity places great demand on bodily metabolism even in brains at rest, sometimes referred to as "dark energy" (Raichle, 2006).

The background activity is characterized by weak correlation and strong desynchronization between individual channels. The overall low background activity level may briefly drop to near zero for some channels, which phenomenon is called "null spike" (Freeman, 2008; Kozma and Freeman, 2008). During null spikes, the analytic phase of the background exhibits sudden changes, jumps, discontinuities; the channels have significant dispersion in their analytic phases. If the background is described as a disordered phase compared to the ordered phase with metastable AM patterns, then the null spikes clearly represent extreme disorder, which we characterize as singularity. The singularity is embedded in the background activity. At the singularity, we observe that the analytic amplitude diminishes and the analytic phase dispersion increases explosively. The very low power of the null spike means that the interactions between neural populations are suppressed. This provides favorable conditions for inputs to have a significant impact on the behavior of neural populations, especially through igniting relevant Hebbian assemblies, which facilitate a consequent rapid propagation of activities.

Null spikes are interpreted as the sites of nucleation initiating a phase transition, following the analogy of crystallization or condensation. For example, when a liquid is converted to a solid phase, the solidification starts as a specific point on the surface, and expands from that point rapidly as the liquid to solid phase transition progresses. Similarly, condensation of steam into the liquid phase starts at a point on the surface; the incipient drop grows from that location by expanding the boundary between the liquid and vapor phases. Following these examples, the initiation of null spike on the cortex may signify the start of the phase transition in the brain dynamics from disorganized phase to organized phase. In brains, the organized phase appears in the form of an emergent AM pattern with increasing power at the frequency of the carrier wave (gamma power).

The synchronized pattern emerges at the wake of a phase gradient rapidly propagating over the surface of the cortex. This phase gradient has the form of a cone and it is called "phase cone" (Freeman, 2004b). Note that there are many phase cones that appear and disappear all the time, however, those phase cones are mostly small (microscopic), and do not grow to the macroscopic size characteristic of a phase transition. Only when the drop of the analytic power coincides with the presence of a suitable stimulus, can we observe the rapid growth of a phase cone to sizes covering large cortical areas. The location of the apex of the cone varies randomly from each burst to the next and has no relation to the stimulus. The conic apex is in itself 
a singularity, and there is some preliminary evidence that its location may correspond to the location of the preceding null spike (Freeman, 2015).

\section{The Collapse of AM Patterns}

AM patterns represent highly organized states of the cortex, which ultimately dissolve through gradual erosion under continual bombardment by sensory stimuli. The collapse of AM patterns can be viewed as a phase transitions from a synchronized to a disorganized state. In physics, such a conversion is described as evaporation of a liquid, or melting of a solid substance. This phase transition requires energy transferred to the system.

AM patterns are synchronized bursts of the activities of large masses of neurons, which emerge through phase transitions initiated by null spikes and exists for a fraction of a second (theta rates). There is a characteristic frequency of the burst in the gamma band due to the interaction of excitatory and inhibitory populations, but there is a marked distribution of frequencies of the myriads of individual feedback loops that contribute to the formation of the AM pattern (Kozma and Freeman, 2008). It is inevitable that variations in these frequencies produce oscillations that become less and less synchronized, thus the collective order of the neural populations decreases. As a result, the overall power of the oscillations diminishes and the AM patterns collapse (Freeman, 2014).

The elimination of the AM pattern drives the dynamics back to the background level, which will produce another AM pattern and the whole cycle starts again. The presence of the continual cycle of the emergence and destruction of metastable AM patterns is an important property of cortical dynamics, which is a lifelong process. In the next section, this cycle is discussed in the context of the cinematic theory of cognition, while energy considerations are described afterwards.

\section{CINEMATIC MODEL OF PERCEPTION AS A SEQUENCE OF PHASE TRANSITIONS}

ECoG measurements with intermittent transitions between synchronized and desynchronized brain states are interpreted in the framework of the cinematic theory of cognition (Freeman, 2007; Kozma and Freeman, 2016). Accordingly, neocortex processes information in frames like a cinema. Metastable AM patterns manifest the "frames," and the phase transitions provide the "shutter" from one frame to the next. Moving from one metastable pattern to the other corresponds to successive images in a movie, which we interpret using the synergetic approach to information processing (Haken, 1983). Haken proposed that state transitions are essential for information transfer between hierarchical levels, by which a collection of particles create an order parameter and in circular causality enslaves the activity of the particles. Cortical AM patterns are the manifestations of the enslavement of individual neural oscillations by collective EEG dynamics (Freeman, 2007).

Figure 3 illustrates the sequential processing in the cinematic model of cognition; the top two diagrams show the superimposed 64 ECoG signals (pass band: $20-28 \mathrm{~Hz}$ ) and the corresponding curves of the analytic power, respectively. The time evolution displays a sequence of beats having relatively high power, separated by periods with diminishing analytic power (marked by blue vertical bars). The duration of a beat is about 100-200 ms, and a metastable AM pattern is sustained during this period. The blue bars correspond to brief time periods of transition from one beat to the other. During the transition, the AM patterns collapse to a singularity (null spike), when the synchrony disappears and the phase relationships exhibit high dispersion.

The cinematic theory employs two main components of cortical dynamics that occur sequentially, namely, the movie frame and the shutter.

- The frames are defined by the metastable AM patterns, which describe a phase with synchronous activity and macroscopic order. The metastable AM patterns represent a transmission mode of operation, i.e., they convey the knowledge contained in the meaning of the stimulus that gave rise to AM patterns. At the ordered phase, the cortex ignores the impact of the irrelevant input stimuli, until the AM pattern finally erodes and leads to the disorganized phase (shutter).

- The shutter is brief ( $20 \mathrm{~ms})$ and it corresponds to the collapse of order due to the desynchronization of the neural activity. This is the receiving phase of the perception cycle, when the analytic power drops near zero and the dynamics becomes susceptible to input stimuli. Once a relevant stimulus is selected, it activates a HCA and induces rapid growth of a large phase cone, which extends over distant cortical areas.

The cinematic theory describes two types of phase transitions, one with the emergence of order from disorder in the form of AM patterns, and the other is the collapse of order manifested in the dissolution of the AM patterns.

- Transition from disorder to order: AM patterns emerge rapidly following the initiation by a null spike under the influence of a relevant stimulus. The large cones are initiated and maintained by corresponding HCAs. These large-scale phase cones enslave the cortical dynamics and lead to the emergence of order in the form of AM patterns. Without activating a HCA, the incipient phase cones cannot grow to macroscopic level, rather they remain localized, and the impact of the input stimuli rapidly fades away.

- Transitions from order to disorder: the degradation of the AM patterns is gradual, under the constant impact of input stimuli. At first, AM patterns are highly synchronized and resist to perturbations in the form of the emergence and collapse of small phase cones during the metastable state. Ultimately, however, the synchrony erodes, the power of the population activity decreases, and the dynamics returns to the disorganized background phase.

The existence of metastable AM patterns and their ultimate collapse can be interpreted in the context of SOC. There are incipient, smaller phase cones during the metastable AM patterns (Freeman, 2004b), which resemble avalanches of various sizes 


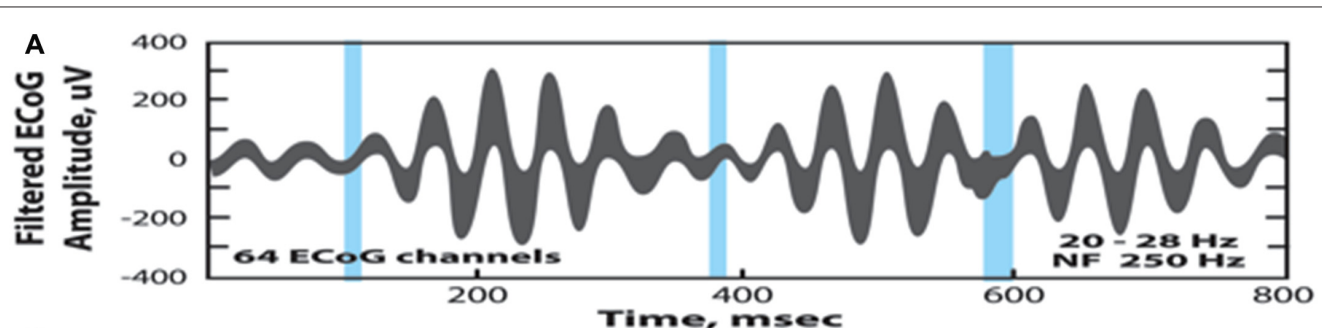

B

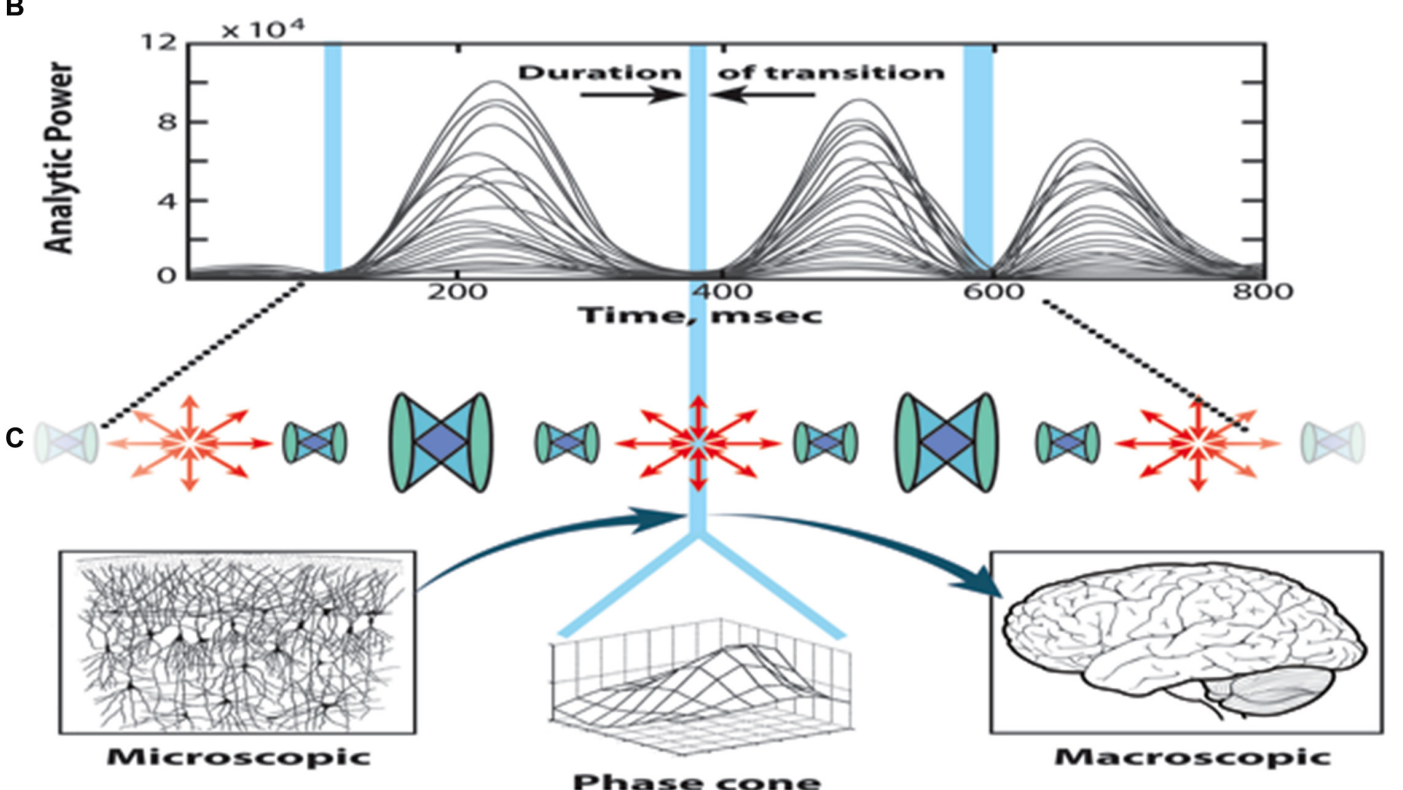

FIGURE 3 | Illustration of the self-organized perception cycle based on the cinematic theory of cognition. (A) Superimposed band-pass filtered ECoG signals. (B) The 64 analytic amplitudes show beats with high amplitudes, interrupted with periods of reduced power, marked by blue bars (null spikes). The high amplitudes between the blue bars correspond to metastable amplitude modulation (AM) patterns carrying the cognitive content (frames). The null spikes are singularities localized in space and time, with high dispersion of the phases (shutter). (C) Following the singularity, large phase cones emerge, which manifest transition from microscopic disorder to macroscopic order (illustration by Chris Gralapp), from Kozma and Freeman (2016).

that maintain the state of SOC (Bak, 1996; Jensen, 1998; Beggs and Plenz, 2003). The power law distribution of avalanche sizes suggests that the neural tissue is in the dynamic state of criticality. These incipient phase cones manifest the dissipation of energy in weak bursts. Such incipient cones may manifest the SOC metastable state, however, they are different from the largescale phase cones emerging during the phase transitions. SOC cannot describe the sequence of transient patterns observed in the perception cycle and described here in the context of the cinematic theory of cognition. Neuropercolation is a suitable mathematical tool to describe cortical phase transitions, as summarized next (Kozma and Puljic, 2015).

\section{DISCUSSION ON GRAPH THEORY INTERPRETATION OF CORTICAL PHASE TRANSITIONS}

The perception cycle is a sequence of transitions between synchronized and desynchronized states. EEG and ECoG measurements provide a window of observation into this cycle by monitoring synchronization properties of the AM patterns.
A prominent example of synchronization-desynchronization transitions in the cortex is depicted in Figure 4, where the analytical phase difference is shown in the vertical axis, against time and space ( $x$ and $y$ axes). Uniformly distributed phase differences indicate synchrony across the array, while highly variable phase differences mark the presence of desynchronization. The upper segment of Figure 4 is based on the $8 \times 8$ array of electrodes with rabbits, while the lower segment is based on intracranial measurements of the EEG of human volunteers using a linear array of 64 electrodes (Freeman, 2004b). One can see extended periods of global synchrony indicated by dominant blue colors, i.e., uniformly low values of phase differences. The periods of synchrony are interrupted by brief desynchronization events shown by a range of colors due to the large spread of the phase differences.

A family of hierarchical models of cortical dynamics has been developed originally for the olfactory system (Freeman, 1979), which is called now Freeman K (Katchalsky) sets. Freeman K sets have been applied as a general neural network model to describe chaotic dynamic memories using encoding of external data in a sequence of spatial oscillatory patterns, mimicking cortical 


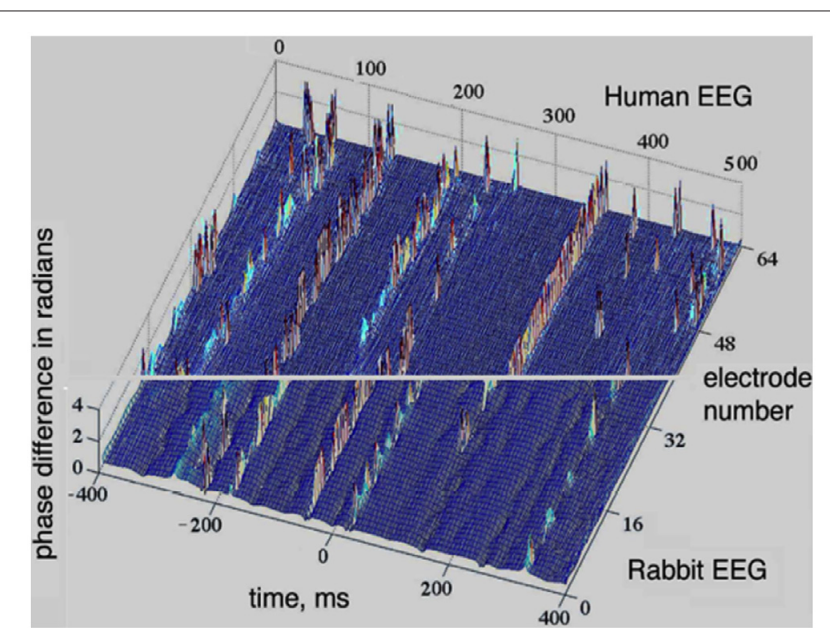

FIGURE 4 | Synchronization-desynchronization effects seen in EEG measurements with humans (lower part) and ECoG with rabbits (upper part); there are extended periods with low phase differences across space (blue color), interrupted by short periods with large phase differences (variable colors). The window of the $8 \times 8$ array was

$5.6 \times 5.6 \mathrm{~mm}$ for the rabbit data (upper half), while a $1 \times 64$ curvilinear array (189 mm long) was used over the scalp of normal human volunteers (lower half; Freeman, 2004b).
AM patterns. The original mathematical formulation of the model was based on a set of second-order ordinary differential equations (ODEs) with distributed parameters (for an overview, see Kozma and Freeman, 2001). Freeman K sets have been used in the past decades for pattern recognition, time series prediction, autonomous navigation and control, and clustering in cybersecurity domains (Harter and Kozma, 2005; Kozma et al., 2007; Freeman and Kozma, 2010; Rosa and Piazentin, 2016).

An alternative implementation of Freeman $\mathrm{K}$ sets uses RGT instead of ODEs and it is called "neuropercolation" (Kozma et al., 2001, 2005; Kozma, 2007). Neuropercolation is based on a mathematical approach combining cellular automata on lattices and random graphs. Neuropercolation considers the interconnected network of neural populations as large-scale random graphs, which exhibit phase transitions near some well-defined critical states. Neuropercolation includes sparse rewiring of connections creating smallworld effects (Watts and Strogatz, 1998), as well as the interaction of excitatory and inhibitory populations (Puljic and Kozma, 2008). It has clear advantages as compared to ODEs in characterizing rapid transients and phase transitions, due to the inherent flexibility of the graph theory framework (Kozma and Puljic, 2013, 2015; Janson et al., 2016).

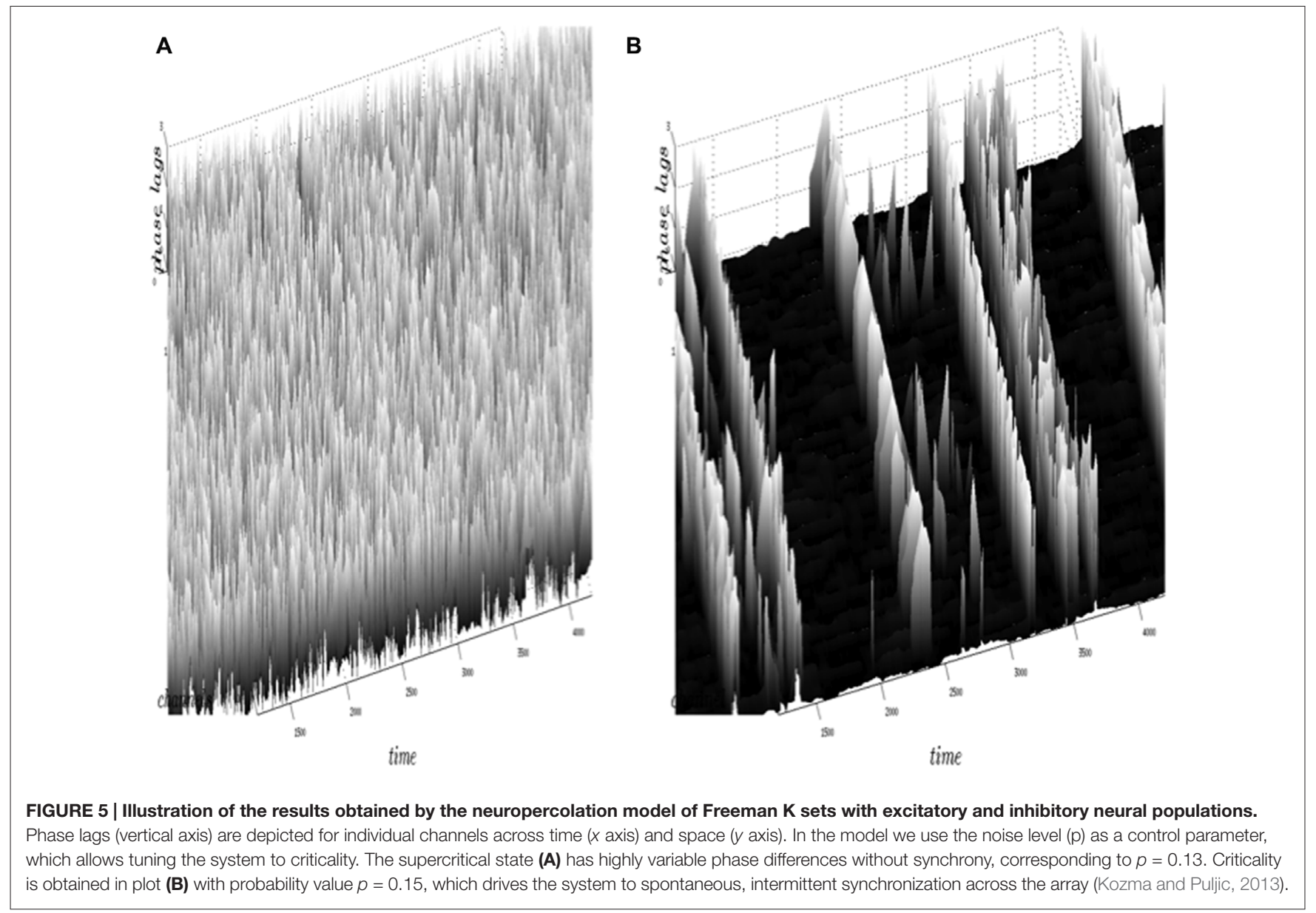


Figure 5 illustrates the results obtained by the neuropercolation model using Freeman $\mathrm{K}$ sets with excitatory and inhibitory populations. Figure $\mathbf{5 A}$ shows the supercritical state with highly variable phase differences (no synchrony), while Figure 5B is an example of the near critical state with intermittent synchronization-desynchronization transitions. The criticality of the system is controlled by the overall noise level ( $\mathrm{p}) ; p=0.13$ belongs to a supercritical state (no synchrony), while $p=0.15$ results in critical state with synchronizationdesynchronization transitions; from Kozma and Puljic (2013). Note that the calculated synchronization-desynchronization transitions across space and time resemble the dynamics observed in measurements with ECoG/EEG arrays. This result supports the hypothesis that the transitions between organized and disorganized phases in the cortex may be the consequence of the cortex residing in a metastable state near criticality.

\section{CONCLUSIONS}

Brains constitute only $2 \%$ of the human body but they use disproportionately high amount of energy (over 20\%), which shows that creating intelligence requires a large amount of metabolic energy. Therefore, energy considerations are very important to understand the nature of biological intelligence in our brains, as well as in attempting to create artificial intelligence in machines.

The cortical energy cycle is summarized as follows, starting from a disordered background state of high entropy and low analytic amplitude. Upon the activation of a HCA by a meaningful stimulus, the synchronized activity of neural populations rapidly propagates across the cortex and creates highly structured AM patterns with low entropy states oscillating in a narrow frequency band (gamma). The formation of AM patterns can be viewed as a condensation process that leads to the dissipation of excess energy in the form of heat that is carried away in the blood stream.

The AM pattern is maintained for some time in a metastable dynamic state that seems to conform to SOC. Synchronized activity of extended neural populations is clearly documented

\section{REFERENCES}

Alagapan, S., Franca, E., Pan, L., Leondopulos, S., Wheeler, B. C., and DeMarse, T. B. (2016). Structure, function, and propagation of information across living two, four, and eight node degree topologies. Front. Bioeng. Biotechnol. 4:15. doi: 10.3389/fbioe.2016. 00015

Amit, D. J. (1995). The Hebbian paradigm reintegrated: local reverberations as internal representations. Behav. Brain Sci. 18, 617-626. doi: 10.1017/s0140525x00040164

Baird, B., Freeman, W. J., Eeckman, F. H., and Yao, Y. (1991). "Applications of chaotic neurodynamics in pattern recognition," in Applications of Artificial Neural Networks II (Int. Society for Optics and Photonics), $12-23$.

Bak, P. (1996). How Nature Works: The Science of Self-Organized Criticality. New York, NY: Copernicus Press.

Beggs, J. M. (2008). The criticality hypothesis: how local cortical networks might optimize information processing. Philos. Trans. A Math. Phys. Eng. Sci. 366, 329-343. doi: 10.1098/rsta.2007.2092 through low phase dispersion between ECoG/EEG channels. Some disturbances in the analytic phase of the cortical tissue appear in the form of small-scale phase cones, which disappear soon after they are formed, obeying the rules of self-similar dynamics of sand piles. The energy released during the formation of the AM pattern is replenished through the metabolism, thus the oxygen debt is repaid (Freeman et al., 2012; Freeman, 2014).

The synchrony represented in the AM pattern is under constant threat by the bombardment of input stimuli and it leads to a degradation of the structure, which can be viewed as an evaporation process. Consequently, the neurons uncouple their dynamics as they are released from the binding represented by the structure. Ultimately, the AM pattern disintegrates, the overall level of firing activity decreases, and the analytic amplitude diminishes. The system returns to a chaotic background state and the cycle is completed (for a detailed description of the cycle, see Kozma and Freeman, 2016).

EEG/ECoG techniques provide insight on the perception cycle in the cortex. Synchronization-desynchronization transitions can be measured by noninvasive scalp EEG (Ruiz et al., 2010; Panagiotides et al., 2011), which allows monitoring the cognitive activity of normal subjects during routine daily activities (Freeman and Quian-Quiroga, 2013). This creates the opportunity to develop various brain-computer interfaces to improve the quality of life of the healthy human population and people with disabilities.

\section{AUTHOR CONTRIBUTIONS}

All authors listed, have made substantial, direct and intellectual contribution to the work, as displayed in the publication.

\section{ACKNOWLEDGMENTS}

This work has been funded in part by National Science Foundation (NSF) CRCNS Program DMS-13-11165, and by Defense Sciences Office, DARPA HR0011-16-1-0006 Superior Artificial Intelligence Project. Fruitful discussions with Joshua Davis, Ray Noack, and Paul Werbos are greatly appreciated.

Beggs, J. M., and Plenz, D. (2003). Neuronal avalanches in neocortical circuits. J. Neurosci. 23, 11167-11177.

Bonachela, J. A., de Franciscis, S., Torres, J. J., and Muñoz, M. A. (2010). Self-organization without conservation: are neuronal avalanches generically critical? J. Stat. Mech. Theory Exp. 2010:P02015. doi: 10.1088/17425468/2010/02/p02015

Bressler, S. L., and Kelso, J. A. S. (2001). Cortical coordination dynamics and cognition. Trends Cogn. Sci. 5, 26-36. doi: 10.1016/s1364-6613(00)01564-3

Bullmore, E., and Sporns, O. (2009). Complex brain networks: graph theoretical analysis of structural and functional systems. Nat. Rev. Neurosci. 10, 186-198. doi: $10.1038 / \mathrm{nrn} 2618$

Bullmore, E., and Sporns, O. (2012). The economy of brain network organization. Nat. Rev. Neurosci. 13, 336-349. doi: 10.1038/nrn3214

Buzsáki, G. (2005). Theta rhythm of navigation: link between path integration and landmark navigation, episodic and semantic memory. Hippocampus 15 , 827-840. doi: 10.1002/hipo.20113

Buzsáki, G. (2015). Hippocampal sharp wave-ripple: a cognitive biomarker for episodic memory and planning. Hippocampus 25, 1073-1188. doi: $10.1002 /$ hipo. 22488 
Buzsáki, G., Anastassiou, C. A., and Koch, C. (2012). The origin of extracellular fields and currents-EEG, ECoG, LFP and spikes. Nat. Rev. Neurosci. 13, 407-420. doi: 10.1038/nrn3241

Canolty, R. T., Ganguly, K., Kennerley, S. W., Cadieu, C. F., Koepsell, K., Wallis, J. D., et al. (2010). Oscillatory phase coupling coordinates anatomically dispersed functional cell assemblies. Proc. Natl. Acad. Sci. U S A 107, 17356-17361. doi: 10.1073/pnas.1008306107

Coombes, S., and Bressloff, P. C. (2005). Bursting: The Genesis of Rhythm in the Nervous System. Singapore: World Scientific.

Crick, F., and Koch, C. (2003). A framework for consciousness. Nat. Neurosci. 6, 119-126. doi: 10.1038/nn0203-119

Davis, J. J. J., Kozma, R., Freeman, W. J. (2013). "Neurophysiological evidence of the cognitive cycle and the emergence of awareness," in International Joint Conference on Awareness Science and Technology \& UbiMedia Computing (iCAST-UMEDIA) (Aizu-Wakamatsu, Japan: IEEE Press), 149-157.

de Arcangelis, L., Lombardi, F., and Herrmann, H. J. (2014). Criticality in the brain. J. Stat. Mech. Theory Exp. 2014:P03026. doi: 10.1088/17425468/2014/03/P03026

Fingelkurts, A. A., Fingelkurts, A. A., and Neves, C. F. (2013). Consciousness as a phenomenon in the operational architectonics of brain organization: criticality and self-organization considerations. Chaos Solitons Fractals 55, 13-31. doi: 10.1016/j.chaos.2013.02.007

Fraiman, D., and Chialvo, D. R. (2012). What kind of noise is brain noise: anomalous scaling behavior of the resting brain activity fluctuations. Front. Physiol. 3:307. doi: 10.3389/fphys.2012.00307

Freeman, W. J. (1975). Mass Action in the Nervous System. New York, NY: Academic Press.

Freeman, W. J. (1979). Nonlinear dynamics of paleocortex manifested in the olfactory EEG. Biol. Cybern. 35, 21-37. doi: 10.1007/bf018 45841

Freeman, W. J. (2004a). Origin, structure, and role of background EEG activity. Part 1. Analytic amplitude. Clin. Neurophysiol. 115, 2077-2088. doi: 10.1016/j. clinph.2004.02.029

Freeman, W. J. (2004b). Origin, structure, and role of background EEG activity. Part 2. Analytic phase. Clin. Neurophysiol. 115, 2089-2107. doi: 10.1016/j. clinph.2004.02.028

Freeman, W. J. (2006). A cinematographic hypothesis of cortical dynamics in perception. Int. J. Psychophysiol. 60, 149-161. doi: 10.1016/j.ijpsycho.2005. 12.009

Freeman, W. J. (2007). “Proposed cortical 'shutter' mechanism in cinematographic perception," in Neurodynamics of Cognition and Consciousness, eds L. Perlovsky and R. Kozma (Heidelberg: Springer), 11-38.

Freeman, W. J. (2008). A pseudo-equilibrium thermodynamic model of information processing in nonlinear brain dynamics. Neural Netw. 21, 257-265. doi: 10.1016/j.neunet.2007.12.011

Freeman, W. J. (2014). "Thermodynamics of cerebral cortex assayed by measures of mass action," in Chaos Information Processing and Paradoxical Game-The Legacy of John S. Nicolis, eds G. Nicolis and V. Basios, (Singapore: World Scientific Publishing Co.), 275-298.

Freeman, W. J. (2015). Mechanism and significance of global coherence in scalp EEG. Curr. Opin. Neurobiol. 31, 199-205. doi: 10.1016/j.conb.2014. 11.008

Freeman, W. J., and Barrie, J. M. (2000). Analysis of spatial patterns of phase in neocortical $\gamma$ EEGs in rabbit. J. Neurophysiol. 84, 1266-1278.

Freeman, W. J., and Holmes, M. D. (2005). Metastability, instability, and state transition in neocortex. Neural Netw. 18, 497-504. doi: 10.1016/j.neunet.2005. 06.014

Freeman, W. J., and Kozma, R. (2010). Freeman's mass action. Scholarpedia 5:8040. doi: 10.4249/scholarpedia. 8040

Freeman, W. J., and Kozma, R., and Vitiello, G. (2012). "Adaptation of the generalized Carnot cycle to describe thermodynamics of cerebral cortex," in The 2012 International Joint Conference Neural Networks (IJCNN) (Brisbane, Australia: IEEE Press), 1-8.

Freeman, W. J., and Quian-Quiroga, R. (2013). Imaging Brain Function with EEG: Advanced Temporal and Spatial Analysis of Electroencephalographic Signals. New York, NY: Springer Verlag.
Freeman, W. J., and Zhai, J. (2009). Simulated power spectral density (PSD) of background electrocorticogram (ECoG). Cogn. Neurodyn. 3, 97-103. doi: 10.1007/s11571-008-9064-y

Friston, K., Breakspear, M., and Deco, D. (2012). Perception and self-organized instability. Front. Comput. Neurosci. 6:44. doi: 10.3389/fncom.2012. 00044

Haimovici, A., Tagliazucchi, E., Balenzuela, P., and Chialvo, D. R. (2013). Brain organization into resting state networks emerges at criticality on a model of the human connectome. Phys. Rev. Lett. 110:178101. doi: 10.1103/physrevlett.110. 178101

Haken, H. (1983). Synergetics: An Introduction. Berlin: Springer-Verlag.

Hansel, D., and Sompolinsky, H. (1992). Synchronization and computation in a chaotic neural network. Phys. Rev. Lett. 68, 718-721. doi: 10.1103/physrevlett. 68.718

Harter, D., and Kozma, R. (2005). Chaotic neurodynamics for autonomous agents. IEEE Trans. Neural Netw. 16, 565-579. doi: 10.1109/tnn.2005. 845086

Hindmarsh, J. L., and Rose, R. M. (1984). A model of neuronal bursting using three coupled first order differential equations. Proc. R. Soc. Lond. B Biol. Sci. 221, 87-102. doi: 10.1098/rspb.1984.0024

Izhikevich, E. M. (2000). Neural excitability, spiking and bursting. Int. J. Bifurcat. Chaos 10, 1171-1266. doi: 10.1142/s0218127400000840

Janson, S., Kozma, R., Ruszinko, M., and Sokolov, Y. (2016). Bootstrap percolation on a random graph coupled with a lattice. arXiv 1507 $07997 \mathrm{v} 2$.

Jensen, H. J. (1998). Self-Organized Criticality: Emergent Complex Behavior in Physical and Biological Systems. New York, NY: Cambridge University Press.

Kozma, R. (1985). Effect of temperature feedback on the neutron-noise field in PWRs. Ann. Nucl. Energy 12, 247-258. doi: 10.1016/0306-4549(85) 90107-0

Kozma, R. (2003). On the constructive role of noise in stabilizing itinerant trajectories on chaotic dynamical systems. Chaos 11, 1078-1090. doi: 10.1063/1. 1599991

Kozma, R. (2007). Neuropercolation. Scholarpedia 2:1360. doi: 10.4249/scholarpedia. 1360

Kozma, R., Aghazarian, H., Huntsberger, T., Tunstel, E., and Freeman, W. J. (2007). Computational aspects of cognition and consciousness in intelligent devices. IEEE Comput. Intell. Mag. 2, 53-64. doi: 10.1109/MCI.2007.385369

Kozma, R., Balister, P., Bollobas, B., and Freeman, W. J. (2001). "Dynamical percolation models of phase transitions in the cortex," in Proc. NOLTA 01 Nonlinear Theory and Applications Symposium (Miyagi, Japan), (Vol. 1) 55-59.

Kozma, R., and Freeman, J. W. (2001). Chaotic resonance: methods and applications for robust classification of noisy and variable patterns. Int. J. Bifurcat. Chaos 10, 2307-2322. doi: 10.1142/s0218127401002870

Kozma, R., and Freeman, W. J. (2008). Intermittent spatio-temporal desynchronization and sequenced synchrony in ECoG signals. Chaos 18:037131. doi: 10.1063/1.2979694

Kozma, R., and Freeman, W. J. (2016). Cognitive Phase Transitions in the Cerebral Cortex-Enhancing the Neuron Doctrine by Modeling Neural Fields. Berlin: Springer Verlag.

Kozma, R., and Puljic, M. (2013). Hierarchical random cellular neural networks for system-level brain-like signal processing. Neural Netw. 45, 101-110. doi: 10.1016/j.neunet.2013.02.010

Kozma, R., and Puljic, M. (2015). Random graph theory and neuropercolation for modeling brain oscillations at criticality. Curr. Opin. Neurobiol. 31, 181-188. doi: 10.1016/j.conb.2014.11.005

Kozma, R., Puljic, M., Balister, P., Bollobas, B., and Freeman, W. J. (2005). Phase transitions in the neuropercolation model of neural populations with mixed local and non-local interactions. Biol. Cybern. 92, 367-379. doi: 10.1007/s00422-005-0565-Z

Lopes-dos-Santos, V., Ribeiro, S., and Tort, A. B. L. (2013). Detecting cell assemblies in large neuronal populations. J. Neurosci. Methods 220, 149-166. doi: 10.1016/j.jneumeth.2013.04.010

March-Leuba, J., and Rey, J. M. (1993). Coupled thermohydraulic neutronic instabilities in boiling water nuclear reactors: a review of the state of the art. Nucl. Eng. Des. 145, 97-111. doi: 10.1016/0029-5493(93) 90061-d 
Palva, J. M., Zhigalov, A., Hirvonen, J., Korhonen, O., Linkenkaer-Hansen, K., and Palva, S. (2013). Neuronal long-range temporal correlations and avalanche dynamics are correlated with behavioral scaling laws. Proc. Natl. Acad. Sci. U S A 110, 3585-3590. doi: 10.1073/pnas.1216855110

Panagiotides, H., Freeman, W. J., Holmes, M. D., and Pantazis, D. (2011). Behavioral states may be associated with distinct spatial patterns in electrocorticogram (ECoG). Cogn. Neurodyn. 5, 55-66. doi: 10.1007/s11571010-9139-4

Petermann, T., Thiagarajan, T. A., Lebedev, M., Nicoleli, M., Chialvo, D. R., and Plenz, D. (2009). Spontaneous cortical activity in awake monkeys composed of neuronal avalanches. Proc. Natl. Acad. Sci. U S A 106, 15921-15926. doi: 10.1073/pnas.0904089106

Plenz, D., and Niebur, E. (Eds). (2014). Criticality in Neural Systems. Hoboken, NJ: John Wiley and Sons.

Puljic, M., and Kozma, R. (2008). Narrow-band oscillations in probabilistic cellular automata. Phys. Rev. E Stat. Nonlin. Soft Matter Phys. 78:026214. doi: 10.1103/physreve.78.026214

Puljic, M., and Kozma, R. (2010). Broad-band oscillations by probabilistic cellular automata. J. Cell. Autom. 5, 491-507.

Pulvermüller, F., and Fadiga, L. (2010). Active perception: sensorimotor circuits as a cortical basis for language. Nat. Rev. Neurosci. 11, 351-360. doi: $10.1038 / \mathrm{nrn} 2811$

Raichle, M. E. (2006). The brain's dark energy. Science 314, 1249-1250. doi: 10.1126/science.\%201134405

Rosa, J. L. G., and Piazentin, D. R. M. (2016). A new cognitive filtering approach based on freeman K3 neural networks. Appl. Intell. 45, 363-382. doi: 10.1007/s10489-016-0772-4

Ruiz, Y., Pockett, S., Freeman, W. J., Gonzales, E., and Li, G. (2010). A method to study global spatial patterns related to sensory perception in scalp EEG. J. Neurosci. Methods 191, 110-118. doi: 10.1016/j.jneumeth.2010. 05.021

Skarda, C. A., and Freeman, W. J. (1987). How brains make chaos in order to make sense of the world. Behav. Brain Sci. 10, 161-195. doi: $10.1017 / \mathrm{s} 0140525 \times 00047336$

Sporns, O. (2013). Structure and function of complex brain networks. Dialogues Clin. Neurosci. 15, 247-262.

Srinivasan, R., Thorpe, S., and Nunez, P. (2013). Top-down influences on local networks: basic theory with experimental implications. Front. Comput. Neurosci. 7:29. doi: 10.3389/fncom.2013.00029

Tagliazucchi, E., and Chialvo, D. R. (2012). Brain complexity born out of criticality. arXiv Preprint arXiv:1211.0309.

Tognoli, E., and Kelso, J. A. S. (2014). The metastable brain. Neuron 81, 35-48. doi: 10.1016/j.neuron.2013.12.022
Tsuda, I. (2001). Towards an interpretation of dynamic neural activity in terms of chaotic dynamical systems. Behav. Brain Sci. 24, 793-810; discussion 810-848. doi: $10.1017 / \mathrm{s} 0140525 \times 01000097$

Turova, T. S. (2012). The emergence of connectivity in neuronal networks: from bootstrap percolation to auto-associative memory. Brain Res. 1434, 277-284. doi: 10.1016/j.brainres.2011.07.050

Upadhyaya, B. R., Kitamura, M., and Kerlin, T. W. (1980). Multivariate signal analysis algorithms for process monitoring and parameter estimation in nuclear reactors. Ann. Nucl. Energy 7, 1-11. doi: 10.1016/0306-4549(80) 90002-x

van Straaten, E. C. W., and Stam, C. J. (2013). Structure out of chaos: functional brain network analysis with EEG, MEG, and functional MRI. Eur. Neuropharmacology 23, 7-18. doi: 10.1016/j.euroneuro.2012.10.010

Watts, D. J., and Strogatz, S. H. (1998). Collective dynamics of 'small-world' networks. Nature 393, 440-442. doi: 10.1038/30918

Wennekers, T., and Palm, G. (2009). Syntactic sequencing in Hebbian cell assemblies. Cogn. Neurodyn. 3, 429-441. doi: 10.1007/s11571-009-9095-z

Werbos, P. J., and Davis, J. J. (2016). Regular cycles of forward and backward signal propagation in prefrontal cortex and in consciousness. Front. Syst. Neurosci. 10:97. doi: 10.3389/fnsys.2016.00097

Yufik, Y. M. (2013). Understanding, consciousness and thermodynamics of cognition. Chaos Solitons Fractals 55, 44-59. doi: 10.1016/j.chaos.2013.04.010

Yufik, Y. M., and Friston, K. (2016). Life and understanding: the origins of "understanding" in self-organizing nervous systems. Front. Syst. Neurosci. 10:98. doi: 10.3389/fnsys.2016.00098

Zalesky, A., Fornito, A., Cocchi, L., Gollo, L. L., and Breakspear, M. (2014). Time-resolved resting-state brain networks. Proc. Natl. Acad. Sci. U S A 111, 10341-10346. doi: 10.1073/pnas.1400181111

Conflict of Interest Statement: The authors declare that the research was conducted in the absence of any commercial or financial relationships that could be construed as a potential conflict of interest.

The reviewer PJW declared a past co-authorship with one of the authors RK to the handling Editor, who ensured that the process met the standards of a fair and objective review.

Copyright (C) 2017 Kozma and Freeman. This is an open-access article distributed under the terms of the Creative Commons Attribution License (CC BY). The use, distribution and reproduction in other forums is permitted, provided the original author(s) or licensor are credited and that the original publication in this journal is cited, in accordance with accepted academic practice. No use, distribution or reproduction is permitted which does not comply with these terms. 